as evidenced by a minority of eligible patients undergoing AS in population based data sets. ${ }^{2,3}$ One of the greatest barriers to improved adoption of AS is the lack of objective selection criteria, which creates significant uncertainty for patients and providers. We agree with the opinions expressed in the letter and recognize that the methodology used to create the DISSRM score is less than ideal for the hypothesis generated. However, the DISSRM score makes the best use of currently available data and readily identifies, in an objective fashion, patients most suitable for AS (ie those who are elderly or have multiple comorbidities, small tumors or poor physical health), those least suitable for AS and those with intermediate criteria.

Use of objective criteria to categorize cases into discrete strata is a starting point for a consultation regarding the management of small renal mass. We do not suggest that stratification by DISSRM score mandates selection of AS or primary intervention, but rather provides an objective system by which patients and providers can understand the risk of death from renal cell carcinoma vs competing causes. The DISSRM score also importantly uses self-reported health related quality of life-an easily acquired metric that may serve as a more practical surrogate for competing risk of death compared to other, more complex health measures.

Neves and Tran appropriately identify lack of systematic use of renal mass biopsy in the DISSRM as the reason for lack of inclusion in the nomogram. Given the limitations of renal mass biopsy (significant nondiagnostic rate and negative predictive value) and its inability to discriminate high grade/risk cT1a tumors from indolent tumors, ${ }^{4}$ we do not favor a "histology based" AS protocol. Rather, we assume and inform these patients that they most likely have an indolent form of renal cell carcinoma and use a more standard protocol for AS. We reserve renal mass biopsy for patients in whom histological data would change clinical treatment, for growing small renal masses and for patients who desire pathological data-approximately $25 \%$ of our cohort. The oncologic outcomes of our observational cohort demonstrate this as a safe approach to AS and justify the criteria associated with selection for $\mathrm{AS}^{5}$

We look forward to further prospective work to better define objective selection criteria, and hope that publication of the DISSRM score will lead to better scoring systems and objective tools to help patients and providers confidently select AS. In doing so we hope to see the expansion of $\mathrm{AS}$ in practices around the world.

1. Patel HD, Semerjian A, Gupta M et al: Surgical removal of renal tumors with low metastatic potential based on clinical radiographic size: a systematic review of the literature. Urol Oncol 2019; 37: 519.

2. Nguyen KA, Nolte AC, Alimi 0 et al: Determinants of active surveillance in patients with small renal masses. Urology 2019; 123: 167.

3. Smaldone MC, Churukanti G, Simhan J et al: Clinical characteristics associated with treatment type for localized renal tumors: implications for practice pattern assessment. Urology 2013; 81: 269.

4. Patel HD, Johnson MH, Pierorazio PM et al: Diagnostic accuracy and risks of biopsy in the diagnosis of a renal mass suspicious for localized renal cell carcinoma: systematic review of the literature. J Urol 2016; 195: 1340.

5. Alam R, Patel HD, Riffon MF et al: Intermediate-term outcomes from the DISSRM registry: a prospective analysis of active surveillance in patients with small renal masses. J Clin Oncol, suppl., 2017; 35: 430.

\title{
Re: Minimally Invasive Radical Prostatectomy after Previous Bladder Outlet Surgery: A Systematic Review and Pooled Analysis of Comparative Studies
}

\author{
A. Veccia, A. Antonelli, S. Francavilla, F. Porpiglia, C. Simeone, E. Lima, \\ H. Zargar, D. Eun, L. J. Hampton and R. Autorino \\ J Urol 2019; 202: 511-517.
}

To the Editor: We read this meta-analysis with great interest. Based on 12 retrospective studies, the authors analyzed the pooled effect of previous bladder outlet surgery (BOS) on minimally invasive radical prostatectomy (RP). They concluded that minimally invasive $\mathrm{RP}$ after previous 
BOS led to higher complication rates and worse functional and oncologic outcomes. Their subgroup analysis indicated that robot-assisted RP could achieve better outcomes. This report deals with an important clinical question. However, some issues may need to be reconsidered.

The authors analyzed studies on laparoscopic and robot-assisted RP after BOS but they did not include open procedures. However, in 2009 Palisaar et al compared outcomes of open RP with and without previous transurethral prostatectomy (TURP) and concluded that open RP after TURP achieved similar perioperative and functional outcomes. ${ }^{1}$ In 2018 Fragkoulis et al did a similar comparison and concluded that open RP after previous TURP was a safe procedure and could achieve equal oncologic and functional outcomes in the hands of experienced surgeons. ${ }^{2} \mathrm{We}$ agree with the conclusion that robot-assisted RP should be preferred over laparoscopic RP regardless of when surgery is needed. However, as a gold standard treatment for localized prostate carcinoma, open RP should always be considered. ${ }^{2}$

Furthermore, time elapsed between BOS and RP is of major importance. Some patients undergo BOS due to benign prostatic hyperplasia and demonstrate prostate cancer after years of followup, ${ }^{3}$ while others undergo BOS and prostate cancer is found incidentally. ${ }^{4}$ Obviously it is a totally different thing to perform RP in these 2 kinds of cases. Taking this into consideration may make the meta-analysis more convincing.

Finally, Teber ${ }^{5}$ and Ramírez Backhaus ${ }^{6}$ et al did research to evaluate the influence of previous TURP on laparoscopic radical prostatectomy in 2009 and 2012, respectively. We fear that these 2 research articles, which met inclusion criteria, were excluded from this meta-analysis without cause.

Respectfully,

\author{
Huihuang Li, Jinbo Chen and Xiongbing Zu \\ Department of Urology \\ Xiangya Hospital, Central South University \\ Changsha, Hunan, China \\ email: zuxbxyyy@126.com; chenjinbo1989@yahoo.com
}

Reply by Authors: As clearly stated, the focus of our review was, indeed, minimally invasive (laparoscopic or robotic) RP. However, we are aware of previous literature on open radical prostatectomy in this setting, and we concur that this remains an option. While length of time between surgery for benign prostatic hyperplasia and surgery for prostate cancer may be a factor impacting the outcome, this possibility could not be assessed in our analysis, which was necessarily limited to retrievable data. Thus, this issue remains to be addressed and demonstrated.

Regarding the studies by Teber ${ }^{5}$ and Ramírez Backhaus ${ }^{6}$ et al, which our colleagues suggest including, we concur that they represent an important contribution to the literature. However, they did not meet predetermined search and inclusion criteria, and for this reason they were not included in the analysis.

1. Palisaar JR, Wenske S, Sommerer F et al: Open radical retropubic prostatectomy gives favourable surgical and functional outcomes after transurethral resection of the prostate. BJU Int 2009; 104: 611.

2. Fragkoulis C, Pappas A, Theocharis $\mathrm{G}$ et al: Open radical prostatectomy after transurethral resection: perioperative, functional, oncologic outcomes. Can J Urol 2018; 25: 9262.

3. Gupta NP, Singh P and Nayyar R: Outcomes of robot-assisted radical prostatectomy in men with previous transurethral resection of prostate. BJU Int 2011; 108: 1501.

4. Bright EA, Manuel C, Goddard JC et al: Incidence and factors predicting the detection of prostate cancer after transurethral resection of the prostate for clinically benign disease. Urol Int 2009; 83: 171. 
5. Teber D, Cresswell J, Ates $\mathrm{M}$ et al: Laparoscopic radical prostatectomy in clinical T1a and T1b prostate cancer: oncologic and functional outcomes—a matched-pair analysis. Urology 2009; 73: 577.

6. Ramírez Backhaus M, Juan Escudero J, Palmero Martí JL et al: Importance of previous transurethral resection of the prostate before laparoscopic radical prostatectomy. Short-term functional outcomes in a single surgeon series. Arch Esp Urol 2012; 65: 675.

\title{
Re: Efficacy and Safety of Complete Intraureteral Stent Placement versus Conventional Stent Placement in Relieving Ureteral Stent Related Symptoms: A Randomized, Prospective, Single Blind, Multicenter Clinical Trial
}

\author{
T. Yoshida, T. Inoue, M. Taguchi, T. Matsuzaki, T. Murota, H. Kinoshita and \\ T. Matsuda
}

J Urol 2019; 202: 164-170

To the Editor: The information in this article is relevant and, as the authors suggest, could be widely and immediately used in clinical practice. There is a long-standing concern regarding symptoms related to indwelling catheters. Also, many drugs have been tested with less than exciting results due to the side effects of anticholinergics, alpha blockers and more recently B3 agonists, or because increased loading of opioids for pain control has not been indicated due to the worldwide opioid abuse epidemic. ${ }^{1,2}$

The fact that the USSQ (Ureteral Stent Symptom Questionnaire), which is specific, could not be used because of the language restriction rendered the work more complex, with a lot of nonspecific data, making this study difficult to compare to others. The USSQ is an instrument that is already used as a standard outcome measure to evaluate the impact of symptoms caused by the indwelling double-pigtail catheter. ${ }^{3}$

Another topic to be considered is the fact that no distal ureter calculus has been evaluated. In theory, the catheter should exceed the area that has been injured by the stone to be able to fulfill the role of drainage of the proximal route, which may not allow it to be completely implanted within the ureter.

Our research group will certainly try to replicate the proposed model. Hopefully we will soon be able to offer more data to validate the use of this model in cases where catheter use is already indispensable.

Respectfully,

Daniel Albrecht Iser, Daisson Trevisol and Betine Pinto Moehlecke Iser

Postgraduate Program in Health Sciences Tubarão University of the South, Santa Catarina Medical School

Santa Catarina, Brazil

1. Volkow ND, Icaza MEM, Poznyak V et al: Addressing the opioid crisis globally. World Psychiatry 2019; 18: 231.

2. Krawczyk N, Greene MC, Zorzanelli R et al: Rising trends of prescription opioid sales in contemporary Brazil, 2009-2015. Am J Public Health 2018; 108: 666 .

3. Joshi HB, Newns N, Stainthorpe A et al: Ureteral Stent Symptom Questionnaire: development and validation of a multidimensional quality of life measure. J Urol 2003; 169: 1060. 